Computer Optics and Nanophotonics

\title{
Asymptotic research in computer optics
}

\author{
Kazanskiy N.L. \\ Image Processing Systems Institute, Russian Academy of Sciences, \\ Samara State Aerospace University
}

\begin{abstract}
I give an overview of the methods and possibilities of the asymptotic studies for solving the computer optics. I analyze the relevance of the use of the results in the design of diffractive optical elements for laser material processing. I discuss the prospects of the developed approaches for the study of the components of diffractive nanophotonics.
\end{abstract}

Keywords: Asymptotic method, diffraction theory, scalar approximation, electromagnetic theory, computer optics, laser technology, diffractive nanophotonics

Citation: Kazanskiy N.L. Asymptotic research in computer optics. Proceedings of Information Technology and Nanotechnology (ITNT-2015), CEUR Workshop Proceedings, 2015; 1490: 151-161. DOI: 10.18287/1613-0073-20151490-151-161

\section{Introduction}

Asymptotic methods have always been in the focus of scientists-opticians [1-3]. These methods have evident interest in recent years [4-11]. Scientists working in the field of diffractive computer optics, also actively used the opportunities provided by asymptotic methods [12-17]. Asymptotic methods are especially good in the study of such class of diffractive optical elements (DOEs), as focusators of laser radiation [1417]. In the paper I give an overview of the methods and possibilities of the asymptotic studies for solving the computer optics. In particular, I analyze the relevance of the use of the results in the design of diffractive optical elements for laser material processing.

\section{Focusators research}

For creating a new focusator we have several important steps: obtaining a phase function; study of the phase function; choice of sampling parameters and method for manufacturing diffractive microrelief; calculation and production of focusator; experimental study of the microrelief and output parameters of focusator. To study the phase function of focusator scientists use analytical calculation of the diffraction patterns of the focused radiation. This calculation must take into account the finite size and specific physical parameters of created focusator [12-17]. Typically, the 
geometric optics approximation is used to calculate the phase function of focusator. Diffractive analysis allows us to explore the limits of this approach. It allowed us to identify the initial values of the physical parameters under which the distortion of the focus area began. This analysis allows us to identify possible errors in the analytical solution of the inverse problem of the diffraction theory.

However, we can carry out diffractive analyzes only for simple phase functions, axially symmetric illuminating beams and focus areas - such as the ring [12], a set of points [18-20], longitudinal [21-24] or cross [15-17, 25-26] segments. In some cases, the analytical study can provide diffractive corrections to the phase function of focusator [14]. Unfortunately, in the framework of the analytical study, we cannot take into account the effect of sampling and quantization of the phase function focusator arising during the manufacture of the DOE. However asymptotic analysis is an important stage of research and complements the capabilities of the computational experiment [21-28].

Unfortunately, asymptotic methods do not allow us to analyze different methods for manufacturing micro-relief of diffractive optical elements [29-36]. Such a study is necessary to select the most appropriate technology for manufacturing DOEs intended for solving a particular problem.

As an example, I cite the results of the asymptotic study of the geometric-optical focusator, concentrated laser beam into the ring $[12,21]$. Fig. 1 shows the results of the asymptotic calculation of the intensity distribution in the focal plane of the geometric-optical focusator into the ring with the following parameters: focal length $f$ $=750 \mathrm{~mm}$; focusator diameter $2 R=25.6 \mathrm{~mm}$; the wavelength of focused beam $\lambda=$ $0.6328 \mu \mathrm{m}$; focal ring diameter $r_{0}=0.1 \mathrm{~mm}$.

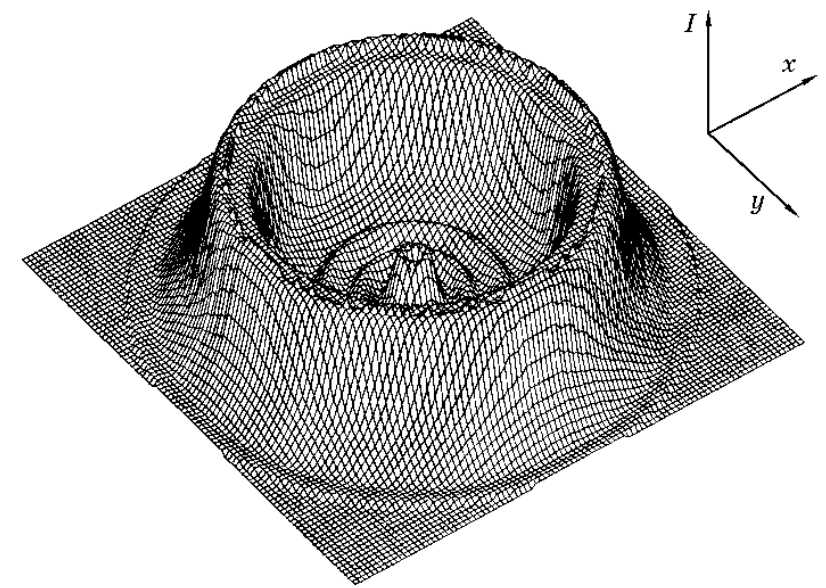

Fig. 1. - The intensity distribution $I(x, y)$ in the focal plane of the focusator into the ring

Asymptotic calculation shows that the diffraction width of the focus ring is comparable to the radius of the ring for focusator with these parameters. As a result, the ring begins to merge with the central spot. The asymptotic calculation shows that such a draining does not occur when the radius of the focal ring in several (and furthermore many) times larger than $0.1 \mathrm{~mm}$. So we clearly see the limitations of the 
methods of geometrical optics in calculating the phase function of focusator. The results can also be used in the study of other types of DOEs [37-45] and to focus the surface electromagnetic waves [46-48].

\section{Temperature calculation}

Asymptotic analysis allows us to optimize the phase function of focusator for use in a specific laser processing technology of a given type of material [49-55]. Focusators have broad prospects for application in a variety of laser materials processing technologies [49-55]: hardening, cutting, welding, drilling, branding, etc. Therefore, the problem of investigating the temperature characteristics of the laser effects produced by focusators is very important. Known focusators form a predetermined intensity distribution in some areas. Laser technology requires forming a desired temperature distribution on the object surface intended for processing. In [26], we conducted an analysis of the temperature distribution formed by focusator into segment (focusator focuses laser light into a line segment located in the focal plane). Asymptotic approach allowed us to obtain a phase function of focusator focusing laser beam into the line segment with a predetermined temperature profile. For example, in [26] we calculated optical element for focusing the circular (and ring) beam into the line segment with a constant temperature distribution. Fig. 2 shows the simulation results for these focusators. Fig. 2a shows a normalized graph of the calculation of temperature distribution along the focal segment for thermal focusator focusing uniform beam of circular cross section of radius $R=5 \mathrm{~mm}$ with the following parameters: the wavelength of the focused radiation $\lambda=1.06 \mu \mathrm{m}$; focal length $f=100 \mathrm{~mm}$; length of focal segment $2 d=2 \mathrm{~mm} ;\left(4 a t_{0}\right)^{1 / 2}=20 \mu \mathrm{m}$ (here $a$ is thermal diffusivity, $t_{0}$ is the duration of the laser action).

Fig. $2 b$ shows the calculated normalized graph of the temperature distribution along the focal segment for the heat focusator focusing uniform beam of annular cross section with radii $R_{l}=3 \mathrm{~mm}$ and $R_{2}=5 \mathrm{~mm}$ with the following parameters: the wavelength of the focused radiation $\lambda=1.06 \mu \mathrm{m}$; focal length $f=400 \mathrm{~mm}$; length of focal segment $2 d=8 \mathrm{~mm}$; $\left(4 a t_{0}\right)^{1 / 2}=0.2 \mathrm{~mm}$ (here used value for thermal diffusivity of the steel $a=12 \mathrm{~mm} / \mathrm{s}^{2}$ ). We can interpret the data in Fig. 2 as a result of forming by the heat focusator a constant temperature profile on the steel surface by the end of the laser pulse duration $t_{0}=0.001 \mathrm{~s}$. For investigated focusators standard deviation from the constant temperature is $8.8 \%$ (for the illuminating beam of circular cross-section, Fig. 2a) and $12.2 \%$ (for the illuminating beam of the annular cross-section, Fig. 2b). It is about two times better than using geometrical optics focusators.

\section{Electromagnetic theory}

In recent years, we are actively developing new asymptotic methods within the electromagnetic theory for calculating the field generated by DOE [56-65].

For example, in [57] we presented an asymptotic method for solving problems of diffraction on the diffractive microrelief. This method combines the geometric-optical approach and solution to the problem of diffraction by a periodic structure with a period comparable to the wavelength. We solved the problem of diffraction by a 
standard quasi-periodic structure that combines the functions of a beam splitter and a diffraction lens. On the basis of the standard solution of the problem we got a simple expression for the field in a plane adjacent to the DOE. The resulting expression allows us to estimate the distribution of the field at the output of the DOE without resorting to complex computational methods.

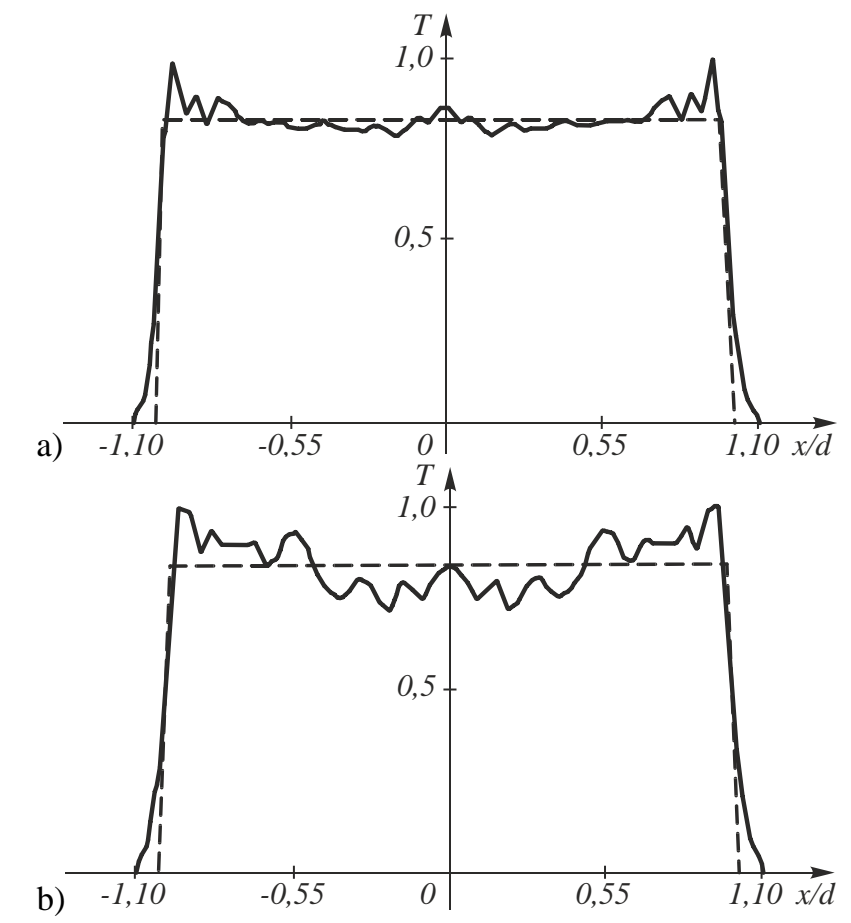

Fig. 2. - The temperature distribution $T$ on the focal segment for thermal focusators: a) uniform illuminating beam with a circular cross-section; $b$ ) uniform illuminating beam with an annular cross-section

We have obtained the results of calculation for the intensity distributions of electromagnetic radiation in the focal plane of the focusator into ring for various combinations of system parameters. Calculation of the field in the focal region, we carried out on the basis of the distribution of the field at the output of the DOE calculated within the electromagnetic theory [57]. Further, the field in the focal plane, we calculated using the propagator, described in [58], on the base of the field at the output of the DOE.

Fig. 3 shows an example for calculation of fields generated by focusators into the ring for the values shown in Table. 1 (all dimensions are in microns). For small relationship $\sigma / f$ ( $\sigma$ is parameter of an illuminating Gaussian beam, $f$ is the focal length), the intensity distribution in the focal plane of focusator into the ring is close to the intensity distributions obtained $[12,21]$ in the framework of scalar approximation. In this case, the energy distribution has good axial symmetry. The 
symmetry is improved in the case of increasing the focal length. The asymmetry in the energy distribution along the ring appears at the increase in the ratio $\sigma / f$. The presence of asymmetry is due to the following factors:

- presence of linear polarization of the incident wave destroys radial symmetry, since the electric fields from different points of the focusators come at different angles in different points of the focal plane;

- in the case of linear polarization of the incident wave the diffractive coefficient depends on the direction of the local grating, it appears with increasing ratio $\sigma / f$.

Table 1. The parameters of focusators

\begin{tabular}{|c|c|c|}
\hline Parameter & $\begin{array}{c}\text { Value (option } \\
1), \mu \mathrm{m}\end{array}$ & $\begin{array}{c}\text { Value (option } \\
2), \mu \mathrm{m}\end{array}$ \\
\hline Wavelength $\lambda$ & 1 & 0.1 \\
\hline $\begin{array}{c}\text { Parameter of } \\
\text { Gaussian beam } \sigma\end{array}$ & 50 & 50 \\
\hline $\begin{array}{c}\text { The distance from } \\
\text { the optical element } \\
\text { to the observation } \\
\text { plane }\end{array}$ & 1000 & 100 \\
\hline Focal length $f$ & 1000 & 100 \\
\hline $\begin{array}{c}\text { Dimensions of the } \\
\text { optical element }\end{array}$ & $500 \times 500$ & $500 \times 500$ \\
\hline
\end{tabular}

Uneven intensity of light in the observation plane of focusator into ring caused unevenness coefficient values in transmission (reflection) of the $E$ - and the $H$ polarization depending on the current value of the period of the band structure (diffraction grating).

\section{Conclusion}

In recent years, scientists were actively developing asymptotic methods in the frame of the electromagnetic theory for calculation of the field formed by the DOEs [56-64]. We use this methods not only for the study of diffractive optical elements (in particular, of short-focus DOEs), but also for the study of nanophotonics components [66-74], for the design of equipment for hyperspectral remote sensing [75-79] and solving other urgent tasks of diffractive nanophotonics [80].

\section{Acknowledgements}

This work was supported by RFBR grants 14-07-00339, 14-07-97008, and fundamental research programs of the Nanotechnology and Information Technology Department of the Russian Academy of Sciences. 

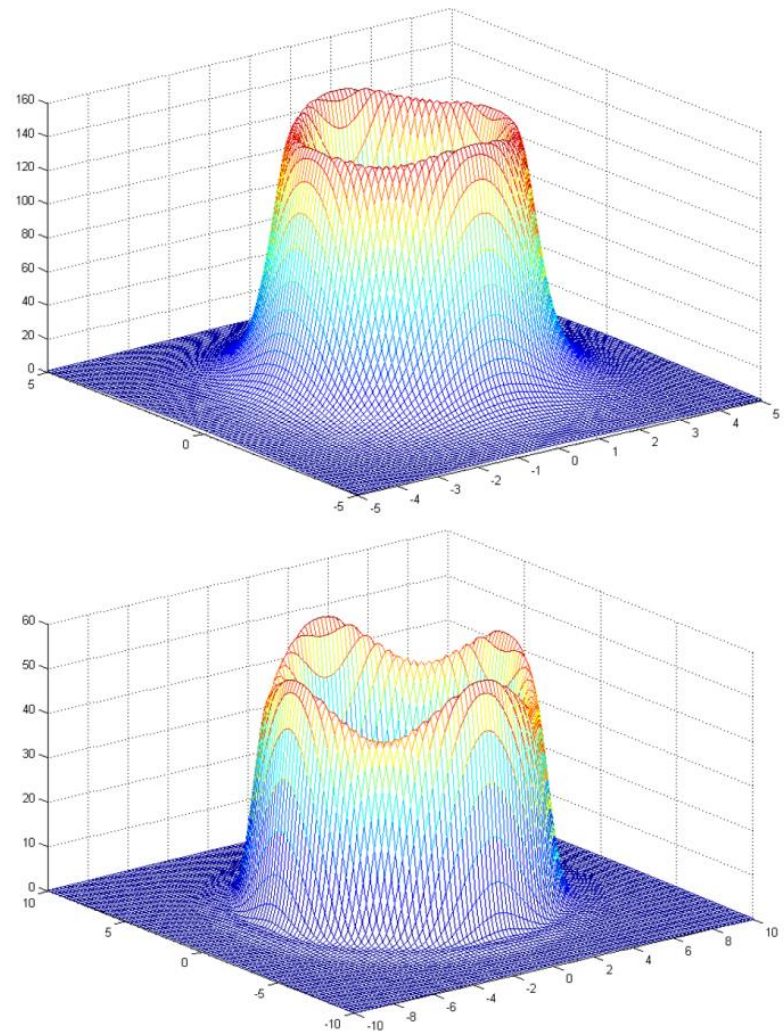

Fig. 3. - The calculated intensity distribution in the focal planes of focusators into the ring with the parameters given in the Table 1 (option 1 - the top; option 2 - the bottom)

\section{References}

1. Walker J. The Analytical Theory of Light. C. J. Clay and Sons, 1904. 432 p.

2. Born M, Wolf E. Principles of Optics 6 ed. New York: Pergamon, 1986.

3. Babich VM, Buldyrev VS. Asymptotic methods in short wave diffraction problems. Moscow: "Nauka" Publisher, 1972. 456 p. [in Russian]

4. Heyman E, Felsen LB. Gaussian beam and pulsed-beam dynamics: complex-source and complex-spectrum formulations within and beyond paraxial asymptotics. J. Opt. Soc. Am. A $2001 ; 18(7): 1588-1611$.

5. Ahrens CD, Ablowitz MJ, Docherty A, Sinkin OV, Grigoryan V, Menyuk CR. Asymptotic analysis of collision-induced timing shifts in return-to-zero quasi-linear systems with predispersion and postdispersion compensation. Optics Letters, 2006; 31(1): 5-7.

6. Simonov AN, Rombach MC. Asymptotic behavior of the spatial frequency response of an optical system with defocus and spherical aberration. J. Opt. Soc. Am. A 2010; 27(12): 2563-2573. 
7. Zhu J, Chen Z, Tang S. Leaky modes of optical waveguides with varied refractive index for microchip optical interconnect applications - asymptotic solutions. Microelectronics Reliability, 2008; 48(4): 555-562.

8. Zhu J, Lu YY. Asymptotic solutions of eigenmodes in slab waveguides terminated by perfectly matched layers. J. Opt. Soc. Amer. A 2013; 30(10): 2090-2095.

9. Sheppard CJR. Limitations of the paraxial Debye approximation. Optics Letters, 2013; 38(7): 1074-1076.

10. Rawlins AD. A note on uniform asymptotic wave diffraction by a wedge. Quarterly Journal of Mechanics and Applied Mathematics, 2014; 67(1): 43-56.

11. Groth SP, Hewett DP, Langdon S. Hybrid numerical-asymptotic approximation for highfrequency scattering by penetrable convex polygons. IMA Journal of Applied Mathematics (Institute of Mathematics and Its Applications), 2015; 80(2): 324-353.

12. Golub MA, Kazanskiy NL, Sisakyan IN, Soifer VA, Kharitonov SI. Diffraction calculation for an optical element which focuses into a ring. Optoelectronics, Instrumentation and Data Processing, 1987; 6: 7-14.

13. Golub MA, Kazanskiy NL, Sisakyan IN, Soifer VA, Kharitonov SI. Diffraction calculation of the field intensity near the focal line of a focuser. Optics and Spectroscopy, 1989; 67(6): 814-815.

14. Golub MA, Doskolovich LL, Kazanskiy NL, Soifer VA, Kharitonov SI. Diffraction approach to the synthesis of multifunctional phase elements. Optics and Spectroscopy, 1992; 73(1): 111-113.

15. Kazanskiy NL, Soifer VA. Diffraction investigation of geometric-optical focusators into segment. Optik - International Journal for Light and Electron Optics, 1994; 96(4): 158162.

16. Kazanskiy NL, Kharitonov SI, Soifer VA. Application of a pseudogeometrical optical approach for calculation of the field formed by a focusator. Optics \& Laser Technology, 1996; 28(4): 297-300.

17. Kazanskiy NL. Research \& Education Center of Diffractive Optics. Proceedings of SPIE, 2012; 8410: 84100R. doi: 10.1117/12.923233.

18. Golub MA, Doskolovich LL, Kazanskiy NL, Kharitonov SI, Soifer VA. Computer generated diffractive multi-focal lens. Journal of Modern Optics, 1992; 39(6): 1245-1251.

19. Doskolovich LL, Kazanskiy NL, Perlo P, Repetto P, Soifer VA. Direct two-dimensional calculation of binary DOEs using a non-binary series expression approach. International Journal of Optoelectronics, 1996; 10(4): 243-249.

20. Kazanskiy N, Skidanov R. Binary beam splitter. Applied Optics, 2012; 51(14): 26722677.

21. Golub MA, Kazanskii NL, Sisakyan IN, Soifer VA. Computational experiment with plane optical elements. Optoelectronics, Instrumentation and Data Processing, 1988; 1: 7889.

22. Kazanskiy NL. Correction of focuser phase function by computer-experimental methods. Computer Optics, 1989; 1(1): 69-73.

23. Kazanskiy NL, Kotlyar VV, Soifer VA. Computer-aided design of diffractive optical elements. Optical Engineering, 1994; 33(10): 3156-3166.

24. Doskolovich LL, Golub MA, Kazanskiy NL, Khramov AG, Pavelyev VS, Seraphimovich PG, Soifer VA, Volotovskiy SG. Software on diffractive optics and computer generated holograms. Proceedings of SPIE, 1995; 2363: 278-284.

25. Doskolovich LL, Kazanskiy NL, Soifer VA. Comparative analysis of different focusators focusing into a segment. Optics and Laser Technology, 1995; 27(4): 207-213. 
26. Doskolovich LL, Kazanskiy NL, Soifer VA, Tzaregorodtzev AYe. Analysis of quasiperiodic and geometric optical solutions of the problem of focusing into an axial segment. Optik - International Journal for Light and Electron Optics, 1995; 101(2): 37-41.

27. Doskolovich LL, Kazanskiy NL, Kharitonov SI, Soifer VA. A method of designing diffractive optical elements focusing into plane areas. Journal of Modern Optics, 1996; 43(7): 1423-1433.

28. Golovashkin DL, Kasanskiy NL. Solving Diffractive Optics Problem using Graphics Processing Units. Optical Memory and Neural Networks (Information Optics), 2011; 20(2): 85-89.

29. Volkov AV, Kazanskiy NL, Moiseev OYu, Soifer VA. A Method for the Diffractive Microrelief Forming Using the Layered Photoresist Growth. Optics and Lasers in Engineering, 1998; 29(4-5): 281-288.

30. Kazanskiy NL, Kolpakov VA, Kolpakov AI. Anisotropic Etching of $\mathrm{SiO}_{2}$ in HighVoltage Gas-Discharge Plasmas. Russian Microelectronics, 2004; 33(3): 169-182.

31. Pavelyev VS, Borodin SA, Kazanskiy NL, Kostyuk GF, Volkov AV. Formation of diffractive microrelief on diamond film surface. Optics \& Laser Technology, 2007; 39(6): 1234-1238.

32. Bezus EA, Doskolovich LL, Kazanskiy NL. Evanescent-wave interferometric nanoscale photolithography using guided-mode resonant gratings. Microelectronic Engineering, 2011; 88(2): 170-174.

33. Bezus EA, Doskolovich LL, Kazanskiy NL. Interference pattern formation in evanescent electromagnetic waves using waveguide diffraction gratings. Quantum Electronics, 2011; 41(8): 759-764.

34. Abulkhanov SR, Kazanskiy NL, Doskolovich LL, Kazakova OY. Manufacture of diffractive optical elements by cutting on numerically controlled machine tools. Russian Engineering Research, 2011; 31(12): 1268-1272.

35. Kazanskiy NL, Kolpakov VA, Podlipnov VV. Gas discharge devices generating the directed fluxes of off-electrode plasma. Vacuum, 2014; 101: 291-297.

36. Volkov AV, Kazanskiy NL, Moiseev OYu, Poletayev SD. Thermal Oxidative Degradation of Molybdenum Films under Laser Ablation. Technical Physics, 2015; 60(2): 265-269. doi: 10.1134/S1063784215020255.

37. Doskolovich LL, Kazanskiy NL, Soifer VA, Kharitonov SI, Perlo P. A DOE to form a line-shaped directivity diagram. Journal of Modern Optics, 2004; 51(13): 1999-2005.

38. Doskolovich LL, Kazanskiy NL, Soifer VA, Perlo P, Repetto P. Design of DOEs for wavelength division and focusing. Journal of Modern Optics, 2005; 52(6): 917-926.

39. Doskolovich LL, Kazanskiy NL, Khonina SN, Skidanov RV, Heikkila N, Siitonen S, Turunen J. Design and investigation of color separation diffraction gratings. Applied Optics, 2007; 46(15): 2825-2830.

40. Karpeev SV, Pavelyev VS, Khonina SN, Kazanskiy NL, Gavrilov AV, Eropolov VA. Fibre sensors based on transverse mode selection. Journal of Modern Optics, 2007; 54(6): 833-844. doi: 10.1080/09500340601066125.

41. Borodin SA, Volkov AV, Kazanskiy NL. Device for analyzing nanoroughness and contamination on a substrate from the dynamic state of a liquid drop deposited on its surface. Journal of Optical Technology, 2009; 76(7): 408-412.

42. Kazanskiy NL, Popov SB. Machine Vision System for Singularity Detection in Monitoring the Long Process. Optical Memory and Neural Networks (Information Optics), 2010; 19(1): 23-30.

43. Khonina SN, Kazanskiy NL, Volotovsky SG. Influence of Vortex Transmission Phase Function on Intensity Distribution in the Focal Area of High-Aperture Focusing System. 
Optical Memory and Neural Networks (Information Optics), 2011; 20(1): 23-42. doi: 10.3103/S1060992X11010024.

44. Aslanov ER, Doskolovich LL, Moiseev MA, Bezus EA, Kazanskiy NL. Design of an optical element forming an axial line segment for efficient LED lighting systems. Optics Express, 2013; 21(23): 28651-28656.

45. Kazanskiy NL, Khonina SN, Skidanov RV, Morozov AA, Kharitonov SI, Volotovskiy SG. Formation of images using multilevel diffractive lens. Computer Optics, 2014; 38(3): 425-434. [in Russian].

46. Bezus EA, Doskolovich LL, Kazanskiy NL. Scattering suppression in plasmonic optics using a simple two-layer dielectric structure. Applied Physics Letters, 2011; 98(22): 221108. doi: 10.1063/1.3597620.

47. Bezus EA, Doskolovich LL, Kazanskiy NL, Soifer VA. Scattering in elements of plasmon optics suppressed by two-layer dielectric structures. Technical Physics Letters, 2011; 37(12): 1091-1095.

48. Bezus EA, Doskolovich LL, Kazanskiy NL. Low-scattering surface plasmon refraction with isotropic materials. Optics Express, 2014; 22(11): 13547-13554. doi: 10.1364/OE.22.013547.

49. Doskolovich LL, Kazanskiy NL, Kharitonov SI, Usplenjev GV. Focusators for laserbranding. Optics and Lasers in Engineering, 1991; 15(5): 311-322.

50. Kazanskiy NL, Murzin SP, Tregub VI. Optical system for realization of selective laser sublimation of metal alloy components. Computer Optics, 2010; 34(4): 481-486. [in Russian]

51. Kazanskiy NL, Murzin SP, Osetrov YeL, Tregub VI. Synthesis of nanoporous structures in metallic materials under laser action. Optics and Lasers in Engineering, 2011; 49(11): 1264-1267.

52. Murzin SP, Tregub VI, Shokova EV, Tregub NV. Thermocycling with pulse-periodic laser action for formation of nanoporous structure in metal material. Computer Optics, 2013; 37(1): 99-105. [in Russian]

53. Murzin SP, Tregub VI, Melnikov AA, Tregub NV. Application of radiation focusators for creation of nanoporous metal materials with high specific surface area by laser action. Computer Optics, 2013; 37(2): 226-233. [in Russian]

54. Murzin SP. Synthesis of metal materials nanoporous structures with cyclic elasto-plastic deformation under laser treatment using radiation focusators. Computer Optics, 2014; 38(2): 249-255. [in Russian]

55. Murzin SP. Method of composite nanomaterials synthesis under metal/oxide pulseperiodic laser treatment. Computer Optics, 2014; 38(3): 469-475. [in Russian]

56. Soifer VA, Kazanskiy NL, Kharitonov SI. Synthesis of a Binary DOE Focusing into an Arbitrary Curve, Using the Electromagnetic Approximation. Optics and Lasers in Engineering, 1998; 29(4-5): 237-247.

57. Kharitonov SI, Doskolovich LL, Kazanskiy NL, Kalyaev ML. The asymptotic method for calculation of the field of the optical elements having the band structure. Computer Optics, 2007; 31(4): 7-18. [in Russian]

58. Kazanskiy NL, Kalyaev ML, Kharitonov SI. Compact recording solutions of Maxwell's equations in the space-frequency representation. Antennas, 2007; 10: 13-21. [in Russian]

59. Khonina SN, Kazanskiy NL, Ustinov AV, Volotovskii SG. The lensacon: nonparaxial effects. Journal of Optical Technology, 2011; 78(11): 724-729.

60. Doskolovich LL, Kazanskiy NL, Kharitonov SI. Integral representations for solutions of Maxwell's equations for anisotropic media. Computer Optics, 2010; 34(1): 52-57. [in Russian] 
61. Kazanskiy NL, Kharitonov SI. Transmission of the space-limited broadband symmetrical radial pulses focused through a thin film. Computer Optics, 2012; 36(1): 4-13. [in Russian]

62. Kazanskiy NL, Khonina SN, Kharitonov SI. The perturbation theory for Schrödinger equation in the periodic environment in momentum representation. Computer Optics, 2012; 36(1): 21-26. [in Russian]

63. Khonina SN, Volotovsky SG, Kharitonov SI, Kazanskiy NL. Calculation of the power spectrum of complex low-dimensional heterostructures in the presence of electric field. Computer Optics, 2012; 36(1): 27-33. [in Russian]

64. Kazanskiy NL, Kharitonov SI, Khonina SN. Joint solution of the Klein-Gordon and Maxwell's equations. Computer Optics, 2012; 36(4): 518-526. [in Russian]

65. Zherdev DA, Kazanskiy NL, Fursov VA, Kharitonov SI. Electromagnetic field scattering simulation from anthropogenic objects on underlying surface. Computer Optics, 2013; 37(1): 91-98. [in Russian]

66. Bykov DA, Doskolovich LL, Soifer VA, Kazanskiy NL. Extraordinary Magneto-Optical Effect of a Change in the Phase of Diffraction Orders in Dielectric Diffraction Gratings. Journal of Experimental and Theoretical Physics, 2010; 111(6): 967-974.

67. Golovashkin DL, Kazanskiy NL. Mesh Domain Decomposition in the Finite-Difference Solution of Maxwell's Equations. Optical Memory \& Neural Networks (Information Optics), 2009; 18(3): 203-211.

68. Kazanskiy NL, Serafimovich PG, Khonina SN. Harnessing the Guided-Mode Resonance to Design Nanooptical Transmission Spectral Filters. Optical Memory and Neural Networks (Information Optics), 2010; 19(4): 318-324. doi: 10.3103/S1060992X10040090.

69. Kazanskiy NL, Serafimovich PG. Cloud Computing for Rigorous Coupled-Wave Analysis. Advances in Optical Technologies, 2012; Article ID 398341. doi: $10.1155 / 2012 / 398341$.

70. Kazanskiy NL, Serafimovich PG, Khonina SN. Use of photonic crystal cavities for temporal differentiation of optical signals. Optics Letters, 2013; 38(7): 1149-1151. doi: 10.1364/OL.38.001149.

71. Kazanskiy NL, Serafimovich PG. Cloud Computing for Nanophotonic Simulations. Lecture Notes in Computer Science, 2013; 7715: 54-67.

72. Kazanskiy NL, Serafimovich PG. Coupled-resonator optical wave-guides for temporal integration of optical signals. Optics Express, 2014; 22(11): 14004-14013. doi: 10.1364/OE.22.014004.

73. Khonina SN, Savelyev DA, Kazanskiy NL. Vortex phase elements as detectors of polarization state. Optics Express, 2015; 23(14): 17845-17859. doi: 10.1364/OE.23.017845.

74. Egorov AV, Kazanskiy NL, Serafimovich PG. Using coupled photonic crystal cavities for increasing of sensor sensitivity. Computer Optics, 2015; 39(2): 158-162. [in Russian]

75. Kazanskiy NL, Kharitonov SI, Khonina SN, Volotovskiy SG, Strelkov YuS. Simulation of hyperspectrometer on spectral linear variable filters. Computer Optics, 2014; 38(2): 256-270. [in Russian]

76. Kazanskiy NL, Kharitonov SI, Karsakov AV, Khonina SN. Modeling action of a hyperspectrometer based on the Offner scheme within geometric optics. Computer Optics, 2014; 38(2): 271-280. [in Russian]

77. Kazanskiy NL, Kharitonov SI, Khonina SN. Simulation of a hyperspectrometer based on linear spectral filters using vector Bessel beams. Computer Optics, 2014; 38(4): 770776. [in Russian]

78. Doskolovich LL, Bezus EA, Bykov DA. On the compensation of the diffraction orders overlap effect in the Offner spectrometer. Computer Optics, 2014; 38(4): 777-782. [in Russian] 
79. Kazanskiy NL, Kharitonov SI, Doskolovich LL, Pavelyev AV. Modeling the performance of a spaceborne hyperspectrometer based on the Offner scheme. Computer Optics, 2015; 39(1). 70-76. [in Russian]

80. Soifer VA. Diffractive Nanophotonics and Advanced Information Technologies. Herald of the Russian Academy of Sciences, 2014; 84(1): 9-18. doi: 10.1134/S1019331614010067. 